Pacific Journal of Mathematics

MAPPINGS AND DECOMPOSITIONS 


\title{
MAPPINGS AND DECOMPOSITIONS
}

\author{
G. K. WILLIAMS
}

If $X$ is a locally connected, locally compact Hausdorff space and $R$ is an equivalence relation on $X$ with fibers which are connected with compact boundaries, then it is known that three types of continuity for $R$ are equivalent. The main result of this note shows that the connectedness of the fibers can be replaced by the requirement that the decomposition be almost proper, i.e., the saturation of each compact set has compact components.

The duality concerning mappings and decompositions is well known [9]. In this note we prove some mapping theorems which result from theorems about decompositions and, vice versa, some theorems on decompositions which follows from mapping theorems. Results concerning quasi-open, proper and almost proper mappings are obtained as well as results on proper and almost proper decompositions.

2. Mappings. A mapping $f: X \rightarrow Y$ is quasi-open if when $y \in Y$ and $U$ is an open set in $X$ which contains a compact component of $f^{-1}(y)$, then $y$ is interior to $f(U)$. It is semiclosed when the image of a compact set is closed. If $f^{-1}(K)$ is compact whenever $K$ is compact, then the mapping is called proper (or compact); if each component of $f^{-1}(K)$ is compact, then $f$ is almost proper. The mapping is monotone if $f^{-1}(y)$ is compact and connected for each $y \in Y$ and it is strongly monotone if the inverse of each compact connected set is compact and connected.

For examples involving quasi-open functions see [8] or [6]. An important application of almost proper mappings is given in [1].

A space is locally peripherally compact if each point has arbitrarily small neighborhoods with compact boundaries. The notation $f: X \Rightarrow Y$ will mean the mapping is onto.

LEMma. If $X$ is a locally peripherally compact Hausdorff space and $f: X \Rightarrow Y$ is a semiclosed quotient mapping with fibers which are connected with compact boundaries, then $f$ is closed and $Y$ is a locally peripherally compact Hausdorff space.

Proof. Let $K \subset X$ be closed and let $y \in Y \backslash f(K)$. Then there is an open set $U$ containing $f^{-1}(y)$ such that bdry $U$ is compact and $U \cap K=\varnothing$. If bdry $U=\varnothing, U$ is an open inverse set, $f(U)$ is open 
and $f(U) \cap f(K)=\varnothing$. If bdry $U \neq \varnothing$, then $C=f($ bdry $U$ ) is closed and if $W=Y \backslash C$ and $D=f^{-1}(W) \cap U$, then $D$ is an open inverse set and hence $f(D)$ is open and $f(D) \cap f(K)=\varnothing$. Thus $f$ is closed.

$Y$ is locally peripherally compact by a result of Stone [7] and is easily seen to be Hausdorff.

REMARK. The fact that $f$ is closed could have been obtained by quoting results from [4], but the above standard argument is more straightforward.

THEOREM 1. If $f: X \Rightarrow Y$ is a monotone mapping where $X, Y$ are Hausdorff and $X$ is locally peripherally compact, then the following are equivalent.

(i) $f$ is a quotient mapping.

(ii) $f$ is closed.

(iii) $f$ is quasi-open.

If, in addition, $Y$ is a $k$-space, then (i), (ii) and (iii) are equivalent to

(iv) $f$ is proper.

Proof. (i) $\rightarrow$ (ii). This is a direct result of the lemma.

(ii) $\rightarrow$ (iii). Let $U$ be an open set containing $f^{-1}(y)$. Then $X \backslash f(X \backslash U)$ is an open set which contains $y$ and which is contained in $f(U)$.

(iii) $\rightarrow$ ( $\mathrm{i}$ ). If $f$ is monotone and quasi-open, then the image of any open inverse set is clearly open.

The last part of the theorem follows from the fact that a mapping is proper if and only if it is closed and has compact point inverses whenever the image space is a $k$-space.

The equivalence of (iii) and (iv) is generalization of a result due to Whyburn [10]. Note also that if any one of the conditions holds, then the inverse of any closed connected set is connected, hence $f$ is strongly monotone.

Using techniques developed by Whyburn we prove the following theorem which will be applied to decompositions in the next section.

THEOREM 2. If $X, Y$ are Hausdorff spaces with $X$ locally compact and locally connected and $f: X \Rightarrow Y$ is a quasi-open almost proper mapping, then $Y$ is locally compact and locally connected and for any region $R \subset Y$ each component of $f^{-1}(R)$ maps onto $R$.

Proof. $f$ is clearly a quotient mapping and hence $Y$ is locally 
connected. The proof that $Y$ is locally compact is also straightforward [5].

To prove the last part of the theorem we first show that it is enough to prove that if $y \in Y$ and $U$ is any open set which contains $y$, there exists a region $R^{*}$ with $y \in R^{*} \subset U$ such that each component of $f^{-1}\left(R^{*}\right)$ maps onto $R^{*}$. For suppose $R$ is any region in $Y$ and $Q$ is a component of $f^{-1}(R)$. Then $f(Q)$ is open because if $z \in f(Q)$ there is a compact component of $f^{-1}(z)$ contained in $Q$ and thus $z$ is interior to $f(Q)$. Now if $f(Q) \neq R$ there is a point

$$
w \in \overline{f(Q)} \cap(R \backslash f(Q)) \text {. }
$$

Let $R^{*} \subset R$ be a region containing $w$ such that each component of $f^{-1}\left(R^{*}\right)$ maps onto $R^{*}$. There exists $w^{\prime} \in f(Q) \cap R^{*}$. Let

$$
x \in Q \cap f^{-1}\left(R^{*}\right)
$$

and let $K$ be the component of $f^{-1}\left(R^{*}\right)$ which contains $x$. Then we must have $K \subset Q$ which contradicts.

Now let $y \in Y$ and suppose $U$ is an open set containing $y$. Let $V$ be a connected open set with compact closure such that $y \in \bar{V} \subset U$ and let $M$ be a component of $f^{-1}(V)$. As before, $f(M)$ is open. Suppose $s \in \overline{f(M)} \cap(V \backslash f(M))$. Then there is a filterbase $A$ on $f(M)$ which converges to $s$. $f^{-1}(A) \cap M$ is a filterbase on $M$ and since $M$ is relatively compact there is a filterbase $B$ subordinate to $f^{-1}(A) \cap M$ such that $B$ converges to a point $x^{\prime}$. But then $f\left(x^{\prime}\right)=s$ and thus $x^{\prime} \in M$ and hence $S \in f(M)$.

3. Decompositions, Let $X$ be a topological space and $R$ an equivalence relation on $X$. Then $X / R$ is the topological space consisting of the set of equivalence classes provided with the quotient topology and $p: X \Rightarrow X / R$ is the natural projection mapping. If $S \subset X$, then $R(S)=\{x \mid x \in X$ and $x R y$ for some $y \in S\}$ is called the saturation of $S$ and, in particular, $R(x)$ is called a fiber.

A relation $R$ is called open (closed) if the saturation of every open (closed) set is open (closed). It is semiclosed if the saturation of every compact set is closed. $R$ is proper if the saturation of each compact set is compact and almost proper if the saturation of each compact set has compact components. $R$ is Hausdorff if the quotient space $X / R$ is Hausdorff.

Three definitions of continuity for decompositions have appeared in the literature and have proved to be useful in analysis as well as topology. (See [11] for a bibliograpy and also for some of the results stated below.) These definitions are as follows:

(1) $R$ is open and closed. 
(2) $R$ is open, semiclosed, and for each region $V \subset X / R$, each component of $p^{-1}(V)$ maps onto $V$.

(3) $R$ is open and Hausdorff.

We shall refer to condition $i, i=1,2,3$, as $C_{i}$ continuity for decompositions.

For general topological spaces these conditions are not related in the sense that one condition always implies another but for locally connected, locally compact Hausdorff spaces we do have that

$$
C_{1} \longrightarrow C_{2} \longrightarrow C_{3}
$$

If $X$ is a locally connected, locally peripherally compact Hausdorff space and the fibers of $R$ are connected with compact boundaries then all three conditions are equivalent. Simple examples show that the connectedness is essential. The question then arises as to what conditions must be satisfied by the decomposition in order to drop the connectedness of the fibers and still have some or all of the $C_{i}$ conditions equivalent. It turns out that the useful notion here is that of an almost proper decomposition.

THEOREM 3. If $X$ is a locally connected, locally compact Hausdorff space and $R$ is an almost proper equivalence relation on $X$, then $C_{2} \leftrightarrow C_{3}$.

Proof. $\rightarrow$ Any $C_{2}$ relation on a locally compact Hausdorff space is $C_{3}$ by [4].

$\leftarrow$ If $R$ is $C_{3}$, then it is open and Hausdorff and hence, open and semiclosed. Thus by Theorem 2 it is enough to show that $p$ is almost proper and to do this we only need to show that if $K$ is a compact set in $X / R$, then there exists a compact set $M$ in $X$ such that $p(M)=K$. For each $x \in p^{-1}(K)$, let $U_{x}$ be a relatively compact open set about $x$. Then there exist $x_{1}, \cdots, x_{n}$ such that $K \subset \bigcup \begin{aligned} & \\ & \end{aligned}\left(U x_{i}\right)$, $i=1, \cdots, n$. Now let $M=\left(\bigcup \bar{U} x_{i} \cap p^{-1}(K)\right)$.

In [1], we stated the following result which is essentially due to Whyburn.

Proposition 1. Let $X$ be a locally connected, locally peripherally compact Hausdorff space and suppose $R$ is a $C_{2}$ relation with fibers which have compact boundaries. Then $R$ is $C_{1}$.

Proposition 1 and Theorem 3 together give us the following result. 
THEOREM 4. If $X$ is a locally connected, locally compact Hausdorff space and $R$ is almost proper with fibers which have compact boundaries then all three continuity conditions are equivalent.

EXAMPLE. Let $S$ be the following subset of the plane,

$$
S=\{(x, y) \mid o \leqq x \leqq 1, y \text { is positive integer }\} \text {. }
$$

Two points are $R$ equivalent if they have the same $x$ coordinate. Then $R$ is an almost proper $C_{3}$ relation, hence it is $C_{2}$ but it is not $C_{1}$. Thus the compactness of the boundaries is essential.

Corollary to THeOREM 4. If $X$ is a connected, locally connected, locally compact Hausdorff space and $R$ is a nondegenerate almost proper $C_{3}$ relation with fibers which have compact boundaries, then $R$ is proper.

In [2], three equivalent conditions were stated concerning proper equivalence relations on locally compact Hausdorff spaces. We observe that it is sufficient for the space to be a $k$-space.

TREOREM 5. If $X$ is a k-space and $R$ is an equivalence relation on $X$, then the following are equivalent.

(i) $R$ is proper.

(ii) Each fiber is compact and has a fundamental system of saturated neighborhoods.

(iii) $X / R$ is a k-space and $p: X \Rightarrow X / R$ is a proper mapping.

Proof. (i) $\rightarrow$ (ii). It is clear that each fiber is compact. We must show that the saturation of each closed set is closed. Let $A \subset X$ be closed and let $C$ be a compact set such that $R(A) \cap C \neq \varnothing$. It suffices to show that $R(A) \cap R(C)$ is closed since

$$
R(A) \cap C=(R(A) \cap R(C)) \cap C .
$$

Consider now $A \cap R(C)$. This is compact and hence $R(A \cap R(C))$ is compact and thus closed. But $R(A \cap R(C))=R(A) \cap R(C)$ and hence $R(A) \cap C$ is closed.

REMARK. This method of proof came out of a conversation with Leonard Rubin. See [3] for another proof of this.

(ii) $\rightarrow$ (iii). Since a quotient space of a $k$-space is a $k$-space if and only if it is Hausdorff we need only show that we can separate points. But we can clearly find disjoint neighborhoods of two distinct 
fibers and thus condition (ii) implies that there must be disjoint saturated neighborhoods of the fibers.

Property (ii) clearly implies that $p$ is closed and since the fibers are compact $p$ is proper.

(iii) $\rightarrow$ (i). Clear.

Another area in which decompositions have been useful is that of the factorization of mappings. It is well known that if $f: X \rightarrow Y$ is a mapping with fibers which have compact components where $X$, $Y$ are Hausdorff and $X$ is locally compact, then $f$ has an essentially unique monotone light factorization where the monotone factor is a quotient mapping. If $X, Y$ are Hausdorff and $f$ is proper, then the same is true. What about the case where $f$ is almost proper and $X$ is a $k$-space or $X$ is locally peripherally compact?

\section{REFERENCES}

1. E. Bishop, Mappings of partially analytic spaces, Amer. J. Math., 83 (1961), 209242.

2. H. Cartan, Quotients of analytic spaces, Cont. to function theory, Tata Inst. Fund. Research, Bombay, 1960.

3. E. Duda, Reflexive compact mappings, Proc. Amer. Math. Soc., 17 (1966), 688-693.

4. J. Flachsmeyer, Über halbstetige Zerlegungen topologischer Räume, Math. Nachr., 24 (1962), 1-12.

5. S. Hanai, On quasi-interior mappings, Math. Japon., 3 (1955), 117-120.

6. K. Stein, Analytische Zerlegung Komplexer Räume, Math. Ann., 132 (1956/57). 6393.

7. A. H. Stone, Metrizability of decomposition spaces, Proc. Amer. Math. Soc., 7(1956), 690-700.

8. C. J. Titus and G. S. Young, The extension of interiority, with some applications, Trans. Amer. Math. Soc., 103 (1962), 382-340.

9. G. T. Whyburn, Continuous decompositions, Amer. J. Math., 71 (1949), 218-226.

10. Open mappings on locally compact spaces, Memoirs of the American Mathematical Society, No. 1, 1950.

11. G. K. Williams, Continuous and proper decompositions, Proc. Amer. Math. Soc., 28 (1971), 267-270.

Received November 11, 1971 and in revised June 9, 1972. Part of this work was completed while the author was a participant in an R.P.C.P. program at the University of Oklahoma sponsored by the National Science Foundation.

SOUTHWESTERN AT MEMPhIS 


\section{PACIFIC JOURNAL OF MATHEMATICS}

EDITORS

\author{
H. SAMELSON \\ Stanford University \\ Stanford, California 94305 \\ C. R. HOBBY \\ University of Washington \\ Seattle, Washington 98105
}

\author{
J. DUGUNDJI \\ Department of Mathematics \\ University of Southern California \\ Los Angeles, California 90007 \\ RICHARD ARENS \\ University of California \\ Los Angeles, California 90024
}

\section{ASSOCIATE EDITORS}

E. F. BECKENBACH

B. H. NeUManN

F. WOLF

K. YosHIDA

\section{SUPPORTING INSTITUTIONS}

UNIVERSITY OF BRITISH COLUMBIA

CALIFORNIA INSTITUTE OF TECHNOLOGY

UNIVERSITY OF CALIFORNIA

MONTANA STATE UNIVERSITY

UNIVERSITY OF NEVADA

NEW MEXICO STATE UNIVERSITY

OREGON STATE UNIVERSITY

UNIVERSITY OF OREGON

OSAKA UNIVERSITY
UNIVERSITY OF SOUTHERN CALIFORNIA

STANFORD UNIVERSITY

UNIVERSITY OF TOKYO

UNIVERSITY OF UTAH

WASHINGTON STATE UNIVERSITY UNIVERSITY OF WASHINGTON

AMERICAN MATHEMATICAL SOCIETY NAVAL WEAPONS CENTER 


\section{Pacific Journal of Mathematics}

\section{Vol. 45, No. $1 \quad$ September, 1973}

William George Bade, Complementation problems for the Baire classes .......... 1

Ian Douglas Brown, Representation of finitely generated nilpotent groups ........ 13

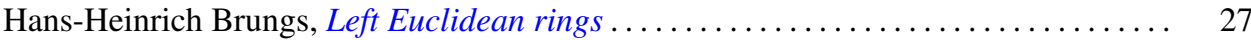

Victor P. Camillo and John Cozzens, A theorem on Noetherian hereditary rings ..... 35

James Cecil Cantrell, Codimension one embeddings of manifolds with locally flat

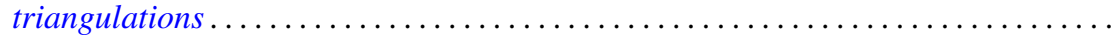

L. Carlitz, Enumeration of up-down permutations by number of rises . . . . . . . . . .

Thomas Ashland Chapman, Surgery and handle straightening in Hilbert cube

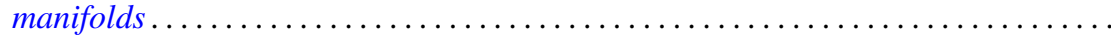

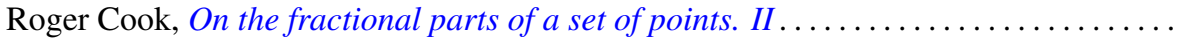

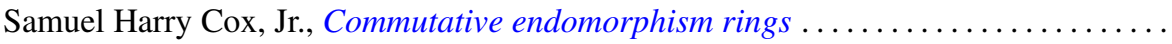

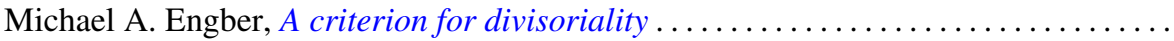

Carl Clifton Faith, When are proper cyclics injective . . . . . . . . . . . . . . 97

David Finkel, Local control and factorization of the focal subgroup . . . . . . . . . 113

Theodore William Gamelin and John Brady Garnett, Bounded approximation by

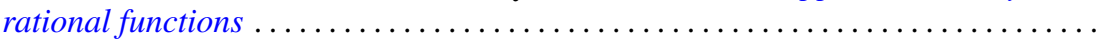

Kazimierz Goebel, On the minimal displacement of points under Lipschitzian

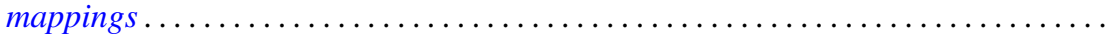

Frederick Paul Greenleaf and Martin Allen Moskowitz, Cyclic vectors for representations associated with positive definite measures: nonseparable

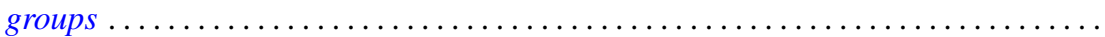

Thomas Guy Hallam and Nelson Onuchic, Asymptotic relations between perturbed linear systems of ordinary differential equations .

David Kent Harrison and Hoyt D. Warner, Infinite primes of fields and completions. .

James Michael Hornell, Divisorial complete intersections . ......

Jan W. Jaworowski, Equivariant extensions of maps ..............

John Jobe, Dendrites, dimension, and the inverse arc function .. .

Gerald William Johnson and David Lee Skoug, Feynman integrals of non-factorable

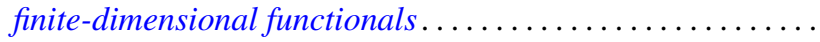

Dong S. Kim, A boundary for the algebras of bounded holomorphic functions ...... 269

Abel Klein, Renormalized products of the generalized free field and its derivatives ... 275

Joseph Michael Lambert, Simultaneous approximation and interpolation in $L_{1}$ and

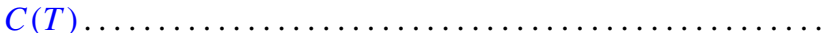

Kelly Denis McKennon, Multipliers of type $(p, p)$ and multipliers of the group

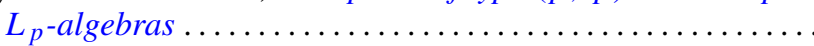

William Charles Nemitz and Thomas Paul Whaley, Varieties of implicative

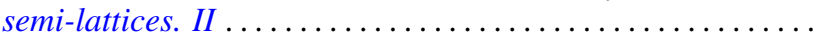

Donald Steven Passman, Some isolated subsets of infinite solvable

Norma Mary Piacun and Li Pi Su, Wallman compactifications on E-completely

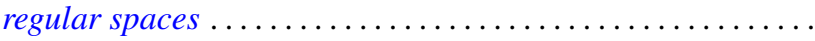

Jack Ray Porter and Charles I. Votaw, $S(\alpha)$ spaces and regular Hausdorff extensions....

Gary Sampson, Two-sided $L_{p}$ estimates of convolution transforms .

Ralph Edwin Showalter, Equations with operators forming a rig
Raymond Earl Smithson, Fixed points in partially ordered sets .

Victor Snaith and John James Ucci, Three remarks on symmetric products and

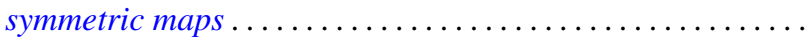

